\title{
Automated, Controlled Deposition of Nanoparticles on Polyelectrolyte-Coated Silicon from Chemomechanically Patterned Droplet Arrays
}

\author{
Jorj I. Owen, ${ }^{\dagger}$ Travis L. Niederhauser, ${ }^{\ddagger}$ Brent A. Wacaser, ${ }^{\dagger}$ \\ Marc P. Christenson, ${ }^{\S}$ Robert C. Davis, ${ }^{\dagger, *}$ and Matthew R. Linford ${ }^{\ddagger *}$ \\ †Department of Physics and Astronomy, Brigham Young University, Provo, UT 84602. \\ Department of Chemistry and Biochemistry, Brigham Young University, Provo, UT 84602. \\ ${ }^{\S}$ Department of Mechanical Engineering, Brigham Young University, Provo, UT 84602.
}

Submitted by Jorj Owen as a capstone research project, to the Department of Physics and Astronomy, April 15, 2004. Under the advisement of Robert Davis.

Also, to be submitted to the surface science journal 'Lab on a Chip'. 


\section{TABLE OF CONTENTS}

Abstract 3

$\begin{array}{ll}\text { Introduction } & 4\end{array}$

$\begin{array}{ll}\text { Experimental } & 7\end{array}$

Results and Discussion $\quad 11$

$\begin{array}{ll}\text { Conclusion } & 21\end{array}$

$\begin{array}{ll}\text { Acknowledgment } & 22\end{array}$

$\begin{array}{ll}\text { References } & 23\end{array}$

$\begin{array}{ll}\text { Appendix } & 25\end{array}$ 


\section{ABSTRACT}

This paper describes three advances in lab-on-a-chip technology. First, it is shown that chemomechanical surface patterning can be performed using a commercially available liquid handler that has undergone only minor modifications. These capabilities are demonstrated by making and then characterizing smaller hydrophobic corrals, made with a diamond tip, than have previously been reported. Hydrophobic corrals are small enclosures on a surface that are ringed by hydrophobic lines. They hold droplets of high surface tension solutions. They allow a surface to be subdivided into individually addressable elements, thus providing a platform for conducting many simultaneous surface experiments with small (down to $c a .1 \mu \mathrm{L}$ ) liquid volumes. An important consequence of this work is that it makes chemomechanical surface patterning, which is a valuable and straightforward method for surface modification, much more accessible to the technical community. Second, it is shown that an entire array of hydrophobic corrals can be simultaneously coated with polyelectrolyte multilayers, but that the hydrophobic corrals still retain the ability to hold liquids after this deposition. The robotic arm of the liquid handler is again employed to manufacture this ultrathin film. Finally, as a demonstration of the capability of this technology to create complex patterned arrays on surfaces from solution for biological or nanostructured materials applications, and again employing the liquid handler, polyelectrolyte-coated hydrophobic corrals are individually addressed and loaded with a solution containing gold nanoparticles for independently specified times. The density and morphology of deposited nanoparticle monolayers were

studied by scanning electron microscopy. The deposition of gold nanoparticles onto a chip occurred at a constant rate $(0.5 \% / \mathrm{min})$ over the range of times studied. 


\section{INTRODUCTION}

The ability to better control and optimize the deposition of nanoparticles, polymers, and small molecules on surfaces will accelerate research and applications in biomolecular, combinatorial, and materials chemistry. To this end we recently developed a new method for simultaneously functionalizing and patterning silicon, ${ }^{1-5}$ which is a material of great importance in modern technology. This method consists of wetting a silicon surface with a reactive compound, and scribing the surface. Scribing activates the silicon so that it reacts with a variety of reagents to form monolayers. The dramatic change in surface wetting that is observed after scribing is powerful evidence for surface functionalization. This change in wetting has been exploited to make checkerboard patterns of hydrophobic lines on silicon. ${ }^{1,5}$ These hydrophobic corrals ${ }^{6}$ hold droplets of water and other liquids with lower surface tensions, ${ }^{1}$ and can even be loaded with different analytes if droplets in the corrals are allowed to dry. ${ }^{5}$

In these original studies, silicon was scribed using a custom apparatus, which consisted of three commercially-available translation stages that were mounted and programmed for scribing. This paper is an important extension of these earlier studies (see Figure 1). First, we show that with minor modification a commercially available liquid handler can be used to create arrays of hydrophobic corrals on silicon surfaces as individual, addressable elements, where we also report the creation and capacities of smaller corrals than were previously made with a diamond tip.

Second, by programming the liquid handler, and by using its robotic arm to hold the patterned silicon, the entire surface can be coated with polyelectrolyte multilayers. ${ }^{7-9}$ In this process the interior regions of hydrophobic corrals are coated, but the hydrophobic 


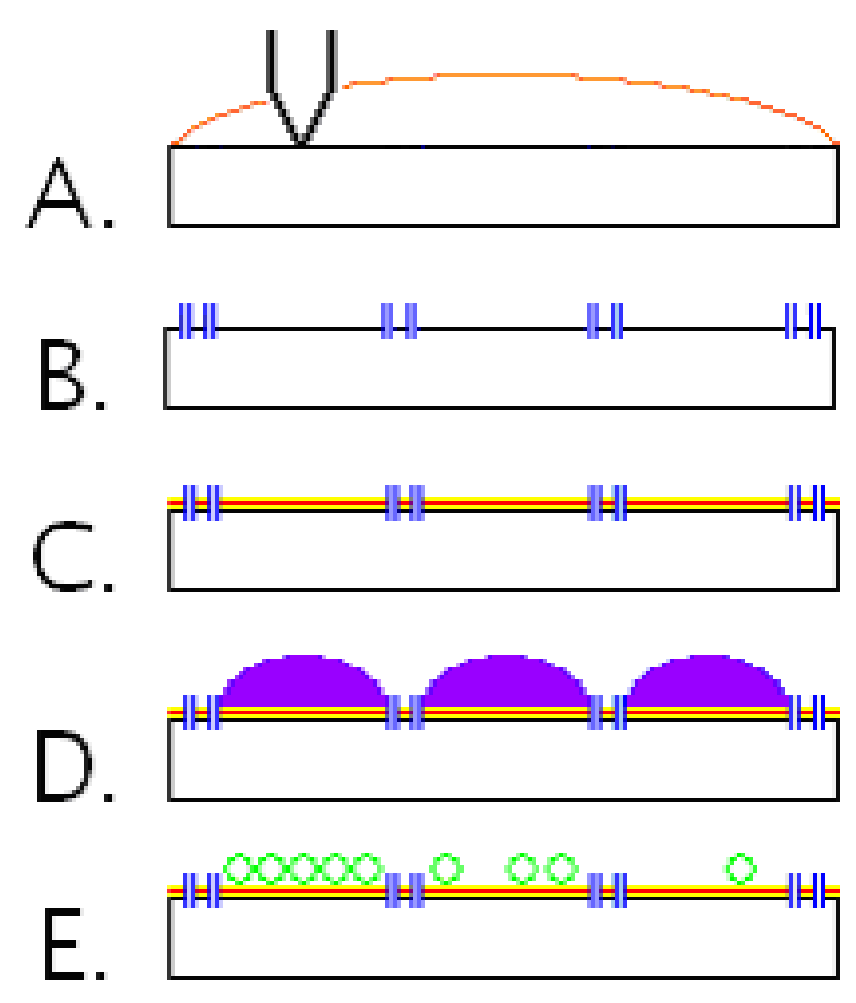

Figure 1: Steps of sample preparation. A) Scribing a Si chip with a diamond tip while it is wet with 1octadecene yields B) hydrophopic lines that separate regions of the silicon chip. C) The entire surface was coated with a polyelectrolyte multilayer. D) A gold nanoparticle solution was deposited in the corrals at different times. E) The finished sample was characterized by electron microscopy. 
corrals retain their ability to hold aqueous solutions. We also report the thickness and roughness of these deposited polyelectrolyte layers.

Finally, as a demonstration of the capabilities of this technology, droplets of a solution of gold nanoparticles, with negatively charged citric acid ligands, were selectively placed on polyelectrolyte-coated corrals at different times to study the kinetics of adsorption of nanoparticles on polyelectrolyte surfaces. Charge driven assembly of nanoparticles on polyelectrolyte surfaces has previously been shown to create layers of disperse particles, islands of aggregates, and dense, close packed layers. ${ }^{10-12}$

Thus, multiple surface reactions/modifications can be simultaneously performed on a single silicon chip using affordable technology. It follows that this method could be used to optimize many different surface processes, e.g., to determine reaction rates and conditions in protein immobilization or in the fabrication of electronic devices such as displays, or to simply localize reagents at different points on surfaces. 


\section{EXPERIMENTAL}

Silicon preparation. Test grade $<100>$ Si wafers were purchased from Montco Silicon Technologies. Prior to use, the Si was cleaned with a 7:3 (v/v) sulfuric acid (conc.) and hydrogen peroxide (30\%) solution for 10 minutes at $c a .100^{\circ} \mathrm{C}$. The wafer was then rinsed thoroughly with deionized (DI) water, and stored in DI water until use.

Chemicals for Scribing Silicon and Polyelectrolyte Multilayers. 1-octadecene (ca. 97\%) was obtained from Fluka. Poly(diallydimethylammonium chloride) (PDADMAC) (20\% in water) with average $M_{w} c a .400,000-500,000$ and poly(sodium 4-styrenesulfonate) (PSS) with average $\mathrm{M}_{\mathrm{w}}$ ca. 70,000 were purchased from Aldrich. Polyelectrolyte solutions were $10 \mathrm{mM}$ in monomer and contained $100 \mathrm{mM} \mathrm{NaCl}$.

Synthesis of Au Nanoparticles. Au nanoparticles were prepared using hydrogen tetrachloroaurate $\left(\mathrm{HAuCl}_{4}\right)\left(99.99 \%\right.$, Alfa Aesar) according to the literature method. ${ }^{13}$ Prior to use all glassware was cleaned with aqua regia (3:1 $\mathrm{HCl}$ (conc.): $\mathrm{HNO}_{3}$ (conc.) $(\mathrm{v} / \mathrm{v}))$, rinsed with triply distilled and filtered water, and dried in an oven. This synthesis produces a stable colloid, which remains in suspension for more than 2 years without sedimentation. The nanoparticle size was $13 \mathrm{~nm}$ as determined by transmission electron microscopy (JEOL 2000FX).

Liquid handler attachments. The robotic arm of a Gilson 215 liquid handler was modified with different commercially available and custom attachments to perform the operations desired in this study. First, the standard needle attachment was replaced with a custom- 
made spring-loaded metal rod that was terminated with a diamond tip and used for scribing. (Capacity measurements on hydrophobic corrals reported in the text are on 5 corrals and measurements were made within one hour of when they were made.) Second, the diamond tipped rod was removed and replaced with a custom-made small metal plate and rod, to which silicon chips were attached with wax. This apparatus was used to dip wafers into the polyelectrolyte solutions. Third, the standard liquid probe, which is a syringe-like accessory for the liquid handler for dispensing liquids, was used to test volume capacities of the corrals, and to deposit Au nanoparticles. DI water was pumped through the liquid probe to remove any air and to clean the needle prior to use. In accord with good practice for this equipment for dispensing solutions, $50 \mu \mathrm{L}$ of air, followed by $300 \mu \mathrm{L}$ of the nanoparticle solution were then drawn into the probe, where the air separates the nanoparticle solution from the DI water in the line, preventing diffusion.

Other Accessories for the Liquid Handler. In order to hold the wafer steady during scribing and Au nanoparticle deposition a vacuum stage was employed. This accessory simply consisted of a flat aluminum block, with appropriate holes drilled in it, which was attached to the house vacuum. To prevent evaporation of the Au nanoparticle solution a water trough was machined into the aluminum block, surrounding the chip platform. A polymethylmethacrylate (PMMA) chamber was made around the vacuum stage. A sliding door at the top of this chamber could be opened and closed by the liquid handler to maintain a high humidity environment during $\mathrm{Au}$ nanoparticle deposition. No noticeable evaporation from droplets of the Au nanoparticle solutions in hydrophobic corrals was observed within an hour in this chamber. 
Between deposition of each polyelectrolyte layer the wafer was moved to a "rinsing station" where any unbound polyelectrolyte was rinsed off with DI water. The rinsing station consists of a PMMA chamber glued together with epoxy, and two small plastic tubes through which DI water flows into and out of the chamber. The flow of water was controlled by a Visual Basic program, which directed the TTL switches on the liquid handler to open and close two solenoid valves at specific times. A rinse consists of filling and draining the chamber four times with water.

Programming the liquid handler. The Gilson 215 liquid handler was controlled by computer via Visual Basic programs. One program directed the robotic arm to make a matrix of hydrophobic corrals of any desired size in $1 \mathrm{~mm}$ increments. A second program directed the arm to deposit the five polyelectrolyte layers. The wafers were dipped for 5 minutes in the polyelectrolyte solutions. A third program directed the deposition of $\mathrm{Au}$ nanoparticles. The program caused the liquid handler to open the sliding lid covering the high humidity chamber, to deposit $15 \mu \mathrm{L}$ of Au nanoparticle solution, and to then close the sliding lid.

Ellipsometry. Both prior to and after polyelectrolyte deposition, film measurements were made with a spectroscopic ellipsometer (M-2000, J.A. Woollam Co.).

Determining percent surface coverage. Images of Au nanoparticles were taken with a FEI XL30 environmental Field Emissions Gun Scanning Electron Microscope (SEM). These images were saved in bitmap file form and converted to black and white images 
using the Scion Image program. White pixels corresponded to Au nanocrystals and black pixels corresponded to the polyelectrolyte coated Si surface. The program counts the number of black and white pixels, thus approximating the percent surface coverage. 


\section{RESULTS AND DISCUSSION}

To show that with minor modification a commercially available liquid handler can be used to create arrays of hydrophobic corrals on silicon surfaces as individual addressable elements, a $2.5 \times 2.5 \mathrm{~cm}^{2} \mathrm{Si}$ chip was mounted on a platform and wet with 1octadecene. The chip was held in place with a vacuum chuck 3 or 4 degrees off axis so that the scribed lines were not parallel to the cleavage planes of the Si chip. The liquid handler's automated arm was fit with a diamond tip, and a $3 \times 3$ pattern of corrals was

scribed. Each corral was $0.5 \times 0.5 \mathrm{~cm}^{2}$ and there was a $1 \mathrm{~mm}$ space between corrals to provided two hydrophobic barriers between them. After scribing, the chip was removed and rinsed thoroughly with acetone, wet with an aqueous $2 \%$ sodium dodecyl sulfate solution, brushed with a soft artists brush or cotton tipped swab, ${ }^{14}$ rinsed with DI water, and dried with a stream of nitrogen gas. This process leaves the chip hydrophilic everywhere except where scribed ${ }^{1}$ (Figure 2).

Using this method, the robotic arm of the liquid handler can be employed to create corrals of various sizes. To demonstrate this capability and to measure the amount of liquid the different sized corrals could hold, nine square corrals ranging from $2 \mathrm{~mm}$ to $10 \mathrm{~mm}$ on a side were created. To measure the maximum capacity of DI water of these corrals, the diamond tip used for scribing was replaced with the liquid probe. This probe was positioned ca. $1 \mathrm{~mm}$ above the chip surface and DI water was then dispensed at a rate of $0.5 \mu \mathrm{L} / 300 \mathrm{~ms}$ until the corrals overflowed. The deposition rate was controlled by computer and halted manually when the corral overflowed. The results from these experiments are plotted in Figure 3, which shows that corral water capacity is linearly dependent on corral area. 


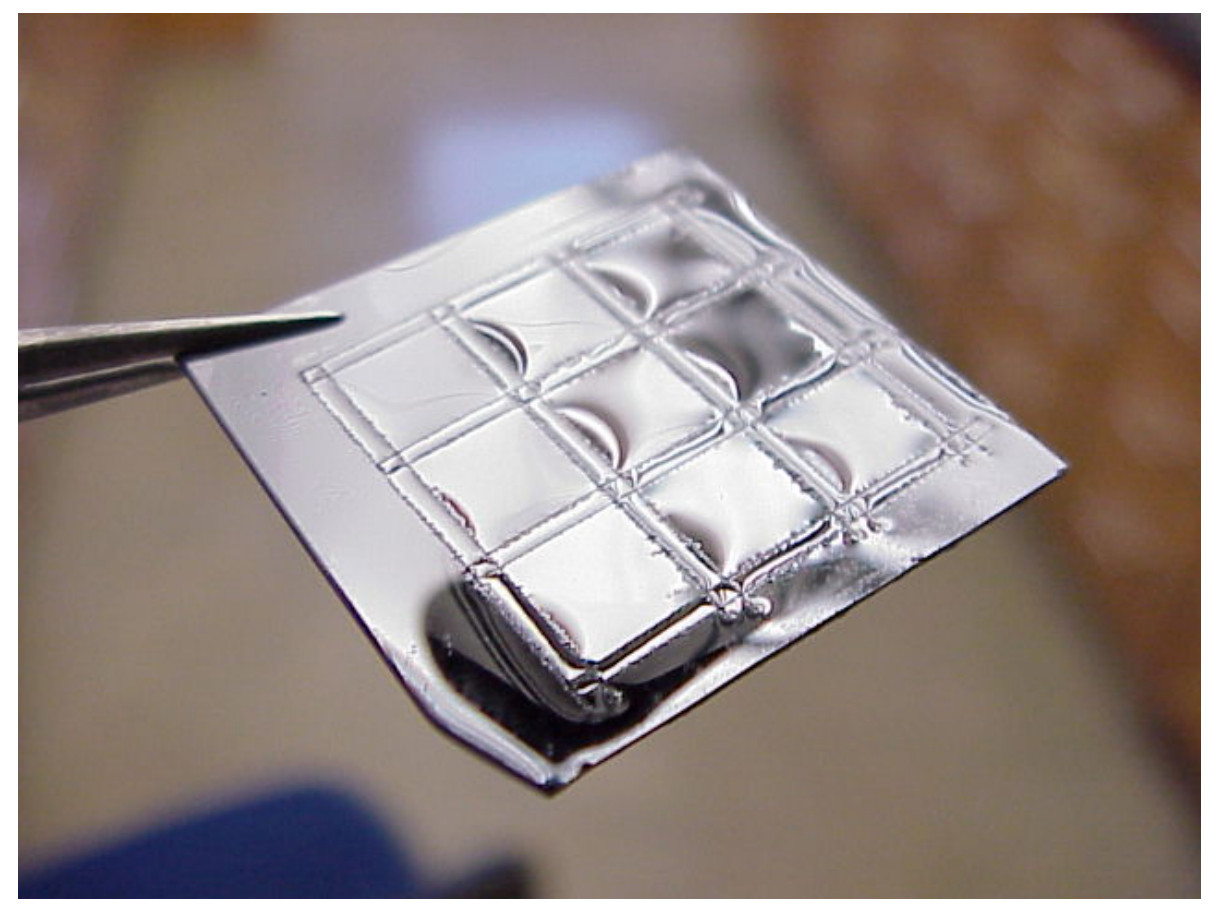

Figure 2: Hydrophilic patches surrounded by a hydrophobic matrix of lines after a rinse with DI water. 


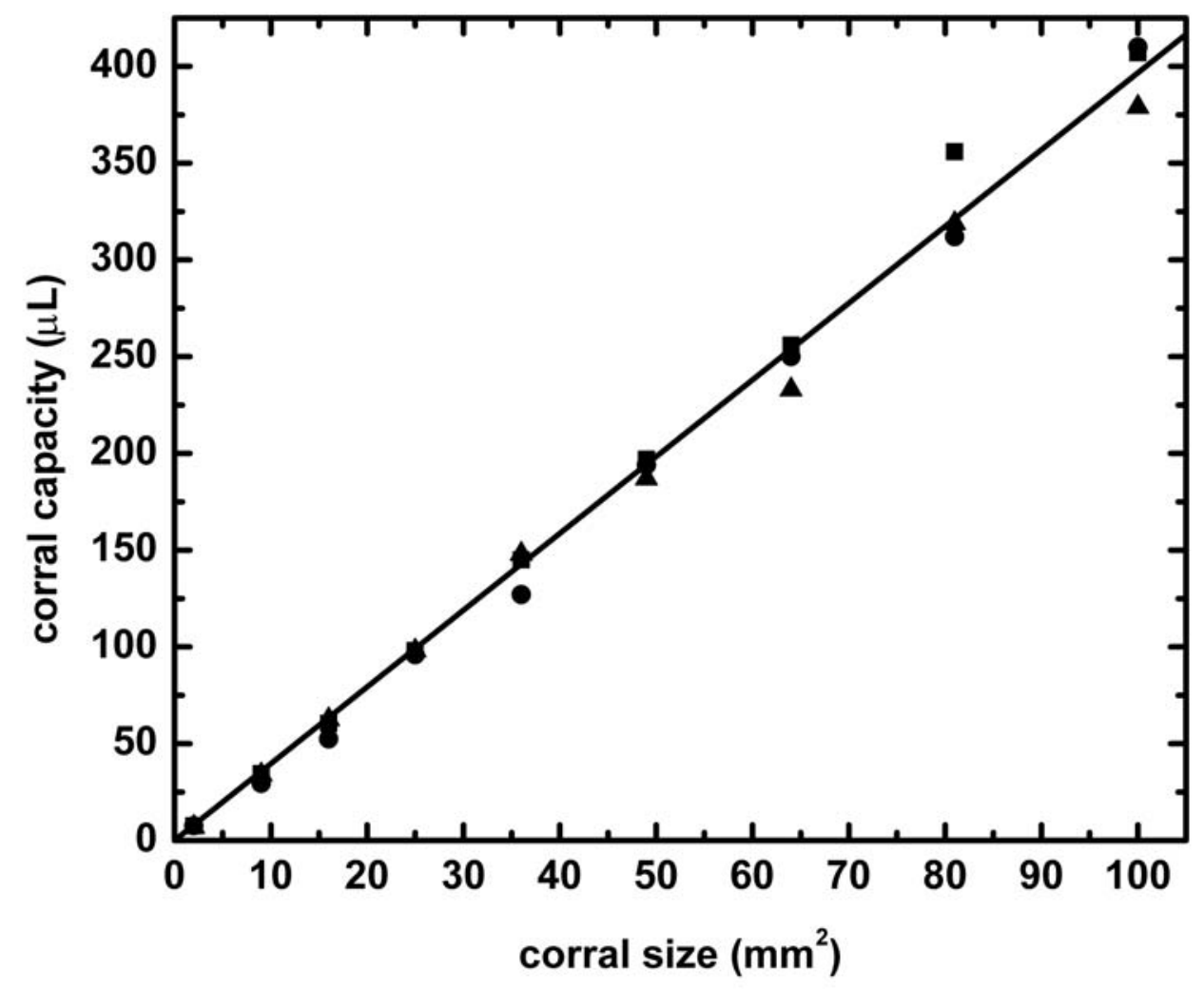

Figure 3: Water capacity of hydrophobic corrals with sides ranging from $2 \mathrm{~mm}$ to $10 \mathrm{~mm}$ in integer increments. 
It was next shown that a silicon surface can be patterned with hydrophobic lines, coated with a polyelectrolyte multilayer, and that hydrophobic corrals still retain their ability to hold aqueous solutions after this surface treatment. It could not be assumed $a$ priori that polyelectrolytes would not coat the lines in hydrophobic corrals and render them useless. The process of coating the patterned silicon with polyelectrolytes by sequentially dipping it into solutions of polycations and polyanions was also automated using with the liquid handler. The silicon chip was attached to the robotic arm of the liquid handler and a Visual BASIC program was executed to direct dipping and rinsing. A total of 5 alternating layers were deposited, starting and ending with PDADMAC (see Figure 4). This method allows simultaneous deposition of polyelectrolyte multilayers in all of the corrals on a surface. In practice, while corral capacity following polyelectrolyte deposition does decrease, sufficient corral capacity is retained for them to function as useful devices. For example, the $25 \mathrm{~mm}^{2}$ corrals initially have a DI water capacity of $80 \pm$ $4 \mu \mathrm{L}$, but their water capacity decreases to $41 \pm 3 \mu \mathrm{L}$ after polyelectrolyte deposition. Part of this decrease is almost certainly due to oxidation of the silicon under the monolayer in the scribed line, which has also been shown to partially reduce hydrophobic corral capacity after long exposure to air or water. ${ }^{15}$

A reasonable explanation for lines to remain hydrophobic after polyelectrolyte deposition on the surrounding silicon is simply their hydrophobicity and lack of surface charge with which they could attract a polyelectrolyte. Alternatively, nanobubbles may be created on the hydrophobic lines when they are submerged in the polyelectrolyte solutions. Nanobubbles have been observed on hydrophobic surfaces via atomic force 


\section{Robotic Arm}
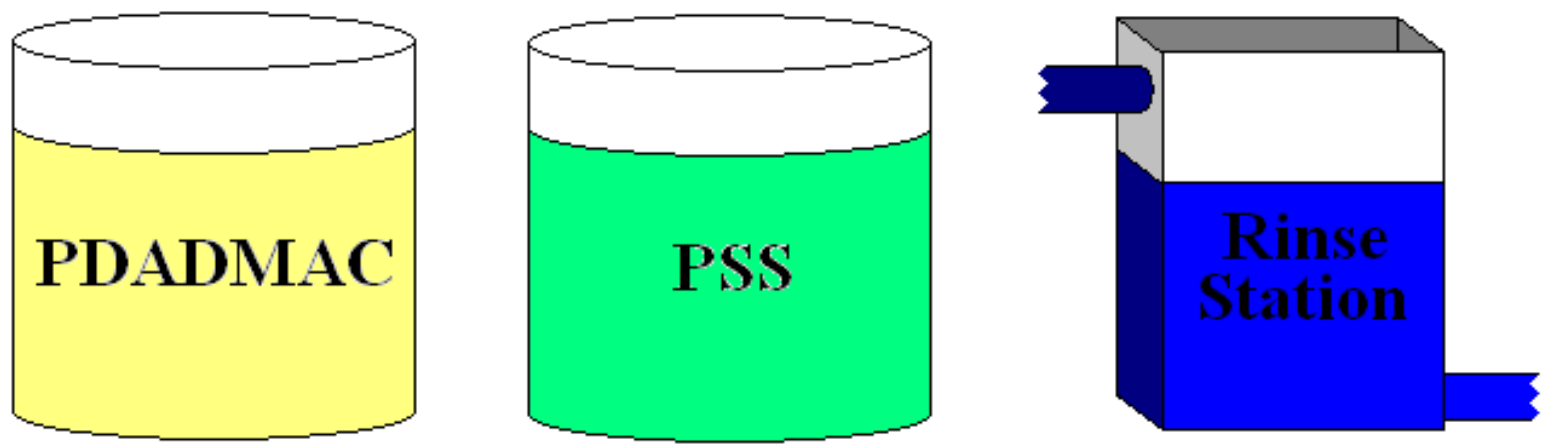

Figure 4: Apparatus for depositing polyelectrolyte multilayers. 
microscopy ${ }^{16}$ and their presence should limit access of the polyelectrolytes to the surface.

To determine the thickness of the deposited multilayers, measurements in each of the nine corrals, and at five different locations in the center corral, were made prior to and after multilayer deposition via spectroscopic ellipsometry using $1 \mathrm{~mm}$ focusing optics. Following the deposition of the PEMs, measurements were taken in approximately the same locations. The data for both sets of measurements were fit using the optical constants of $\mathrm{SiO}_{2}$, as most organics and $\mathrm{SiO}_{2}$ have similar optical properties, ${ }^{17,18}$ and ellipsometry is relatively insensitive to changes in optical constants of very thin films $(<50 \AA) .{ }^{19}$ The thickness of the native oxide was subtracted from the polyelectrolyte-SiO $\mathrm{S}_{2}$ thickness to give the multilayer thickness. The layers were $c a .3 \mathrm{~nm}$ thick, varying by $<$ $0.3 \mathrm{~nm}$ within a chip and by $<0.5 \mathrm{~nm}$ from chip to chip. Table 1 shows the excellent reproducibility of this method.

It is envisioned that hydrophobic corrals with different surface chemistries and coatings could have a number of interesting uses. As a demonstration of these possibilities a solution of Au nanoparticles was dispensed into the corrals at different times to study the kinetics of nanoparticle deposition on a polyelectrolyte multilayer. The Au nanoparticles were terminated with citric acid ligands, which gave them a negative charge. This procedure was performed entirely under computer control using the liquid probe on the liquid handler. For these experiments the chip was placed within a high humidity chamber and held in position with a vacuum chuck. $15 \mu \mathrm{L}$ of nanoparticle solution (notice that this is significantly less than the corral capacity of $c a .41 \mu \mathrm{L}$ ) was dispensed into 8 of 9 corrals (in a $3 \times 3$ pattern) at specified times within an hour. Before 


\begin{tabular}{|c|c|c|c|}
\hline $\begin{array}{l}\text { Chip } \\
\text { number }\end{array}$ & $\begin{array}{l}\text { Thickness of the } \\
\mathrm{SiO}_{2} \text { (in nm). }\end{array}$ & $\begin{array}{l}\text { Thickness of the } \mathrm{SiO}_{2} \text { and } \\
\text { PEM's (in nm). }\end{array}$ & $\begin{array}{l}\text { Thickness of the } \\
\text { PEM's (in nm). }\end{array}$ \\
\hline 1 & $2.04 \pm 0.03$ & $5.5 \pm 0.2$ & $3.5 \pm 0.2$ \\
\hline 2 & $1.94 \pm 0.02$ & $5.4 \pm 0.1$ & $3.5 \pm 0.1$ \\
\hline 3 & $1.82 \pm 0.05$ & $5.6 \pm 0.3$ & $3.7 \pm 0.3$ \\
\hline 4 & $2.0 \pm 0.05$ & $5.5 \pm 0.2$ & $3.5 \pm 0.2$ \\
\hline 5 & $2.30 \pm 0.07$ & $5.5 \pm 0.1$ & $3.2 \pm 0.1$ \\
\hline
\end{tabular}

The errors are the standard deviations of the measurements.

Table 1: Average film thickness plus or minus the standard deviation of 13 measurements made on each chip. 
adding an aliquot of solution to a corral, the probe pushed open the sliding door on the chamber. After an aliquot was added to a corral, the probe pushed the sliding door shut to prevent evaporation of the nanoparticle solution. Depositions were made so that the corrals were exposed for $60,50,40,30,20,10,5$, and 2 minutes. After 60 minutes, the chip was rinsed manually by dipping it in DI water. Deposition and rinsing times are accurate to within $2.5 \%$.

To determine the coverage of nanoparticles, the sample was examined by SEM. Electron micrographs of the interior of a corral that was exposed to the Au nanoparticle solution for 10 minutes and 60 minutes are shown in Figure 5. In most locations in the nanoparticles-treated corrals, the nanoparticles appear to be in a single layer, however in some locations, such as at the top of Figure 5(B), there are particles lying on top of each other.

SEM images were processed using image analysis software, which provided an estimate of surface coverage with gold nanoparticles. Figure 6 shows a plot of surface coverage versus exposure time to nanoparticle solution. Over the range from 2 to $60 \mathrm{~min}$, the surface coverage versus exposure time appears to be linear within the range of experimental error. Interestingly, the fitted line has a y-intercept near $6 \%$, which suggests some rapid deposition at first that occurs either at the initial droplet contact or within the first two minutes of exposure to the nanoparticle solution. This rapid deposition is followed by a steady deposition rate of $c a .0 .5 \%$ per minute. 

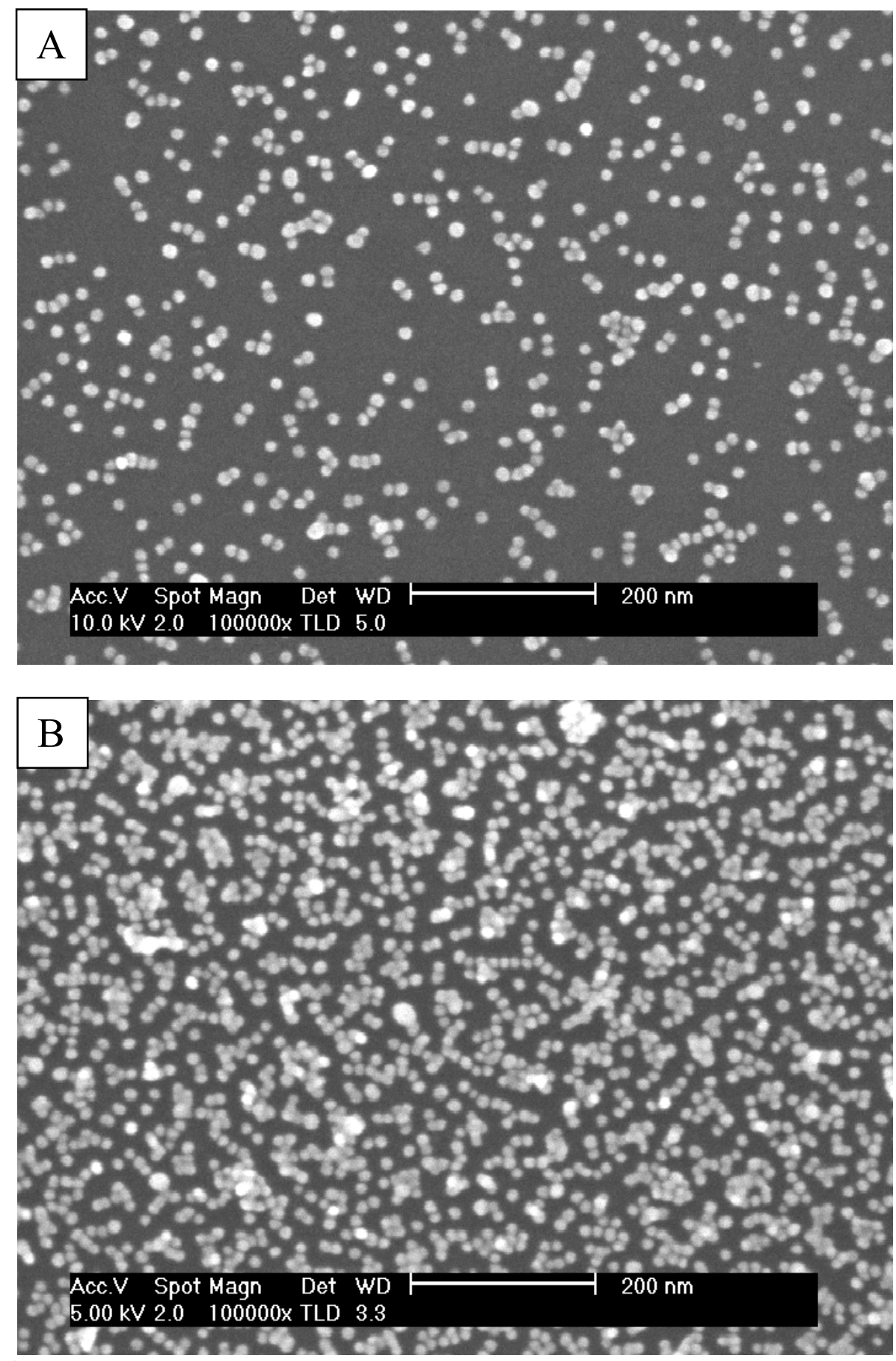

Figure 5: SEM image of sample after exposure to Au nanoparticle solution for A) 10 minutes, and B) 60 minutes. Scale bars are $200 \mathrm{~nm}$. 


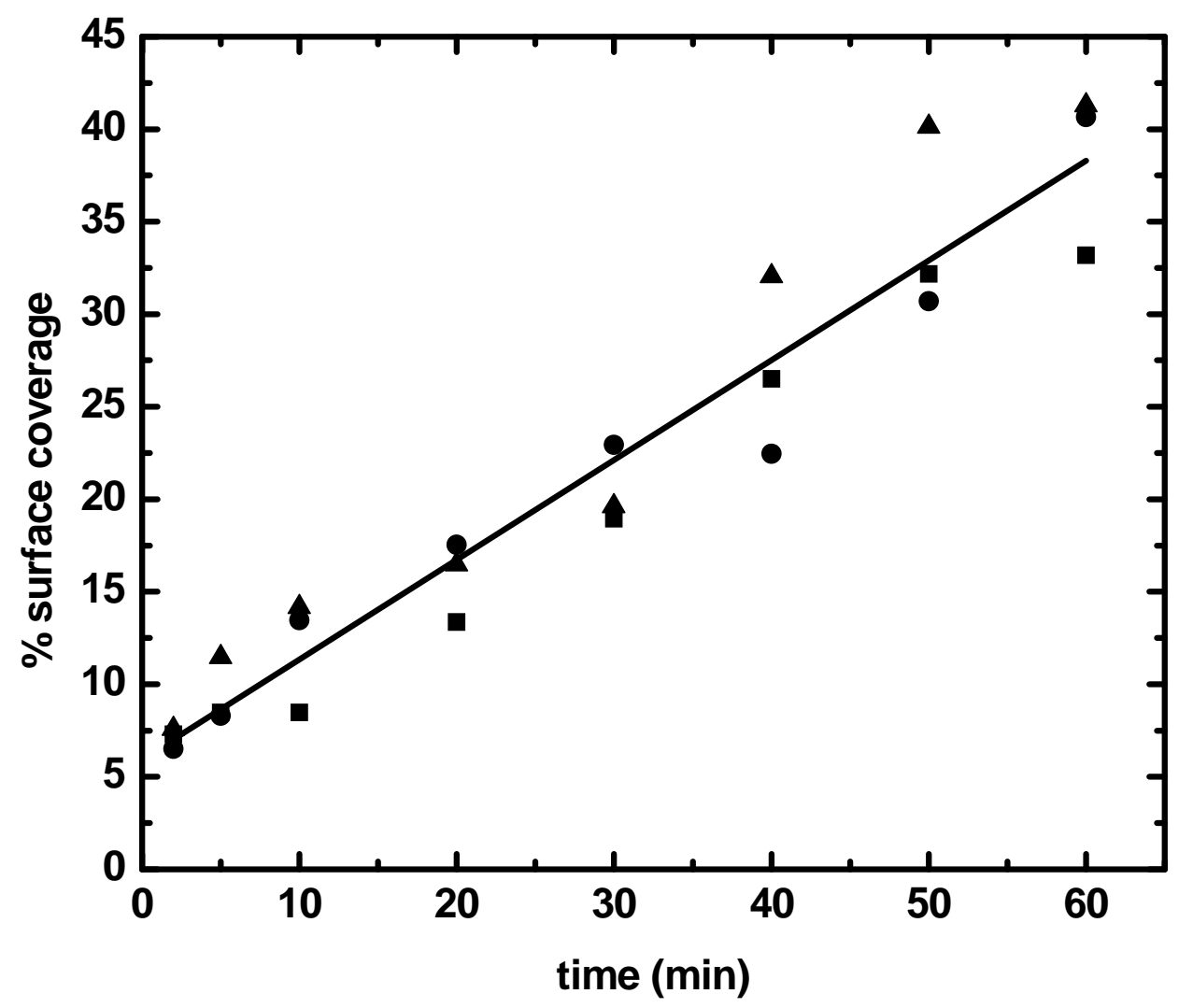

Figure 6: Surface coverage of gold nanoparticles as a function of exposure time to a solution of gold nanoparticles. Symbols represent different experiments on different chips. The solid line is a linear least squares fit to the data. 


\section{CONCLUSION}

This paper shows a method for inexpensively preparing wafers for performing multiple surface experiments on a single chip. Using a commercially available Liquid Handler, silicon was chemomechanically patterned with hydrophobic lines, and coated with PEMs. The kinetics of Au nanoparticle deposition from microliter droplets onto this surface was then determined. All these steps were done under computer control resulting in precise line placement, dipping and rinsing times, and droplet volume and contact time. Control of these variables improves experimental reproducibility.

We are far from the limits of corral density achievable with chemomechanical patterning. Thus, it should be possible to use this method to perform many more experiments on a single chip than were demonstrated in this study, allowing smaller volumes of expensive reagents, such as biomolecules, to be employed, and significantly increasing the amount of information that can be derived from the system. For example, chemomechanical pattering can be performed using an atomic force microscope and linewidths of $<50 \mathrm{~nm}$ are routinely achieved. ${ }^{20}$ Thus, the techniques described in this paper could very well play a role in a solution-based deposition of complex functional materials from nanoscale components at high lateral resolution. 


\section{ACKNOWLEDMENTS}

MRL and RCD thank Gilson, Inc. for loaning the 215 liquid handler. They also acknowledge the Petroleum Research Fund, the Research Corporation, the Department of Chemistry and Biochemistry and the Department of Physics and Astronomy at BYU for support for this research. 


\section{REFERENCES}

(1) Niederhauser, T. L.; Jiang, G.; Lua, Y.-Y.; Dorff, M. J.; Woolley, A. T.; Asplund, M. C.; Berges, D. A.; Linford, M. R. Preparing Monolayers on Silicon and Patterning Silicon Surfaces under Ambient Conditions by Scribing in the Presence of Reactive Species. Langmuir 2001, 17, 5889-5900.

(2) Niederhauser, T. L.; Lua, Y.-Y.; Jiang, G.; Davis, S. D.; Matheson, R.; Hess, D. A.; Mowat, I. A.; Linford, M. R. Arrays of Chemomechanically Patterned Patches of Homogeneous and Mixed Monolayers of 1-Alkenes and Alcohols on Single Silicon Surfaces. Angew.Chem., Int.Ed.Engl. 2002, 13, 2353-2356.

(3) Niederhauser, T. L.; Lua, Y.-Y.; Sun, Y.; Jiang, G.; Strossman, G. S.; Pianetta, P.; Linford, M. R. Formation of (Functionalized) Monolayers and Simultaneous Surface Patterning by Scribing Silicon in the Presence of Alkyl Halides. Chemistry of Materials 2002, 14, 27-29.

(4) Lua, Y.-Y.; Lee, M. V.; Fillmore, W. J. J.; Matheson, R.; Sathyapalan, A.; Asplund, M. C.; Fleming, S. A.; Linford, M. R. Amine-Reactive Monolayers on Scribed Silicon with Controlled Levels of Functionality: Reaction of a Bare Silicon Surface with Mono- and Diepoxides. Angew.Chem.Int.Ed.Eng. 2003, 42, 4046-4049.

(5) Lua, Y.-Y.; Niederhauser, T. L.; Wacaser, B. A.; Mowat, I. A.; Woolley, A. T.; Davis, R. C.; Fishman, H. A.; Linford, M. R. Chemomechanical Production of Sub- $\mu \mathrm{m}$ Edge Width, Functionalized, $20 \mu \mathrm{m}$ Features on Silicon. Langmuir 2003, 19, 985-988.

(6) Abbott, N. L.; Whitesides, G. M.; Racz, L. M.; Szekely, J. Using Finite Element Analysis To Calculate the Shapes of Geometrically Confined Drops of Liquids on Patterned, Self-Assembled Monolayers: A New Method To Estimate Excess Interfacial Free Energies gsv-gsl. Journal of the American Chemical Society 1994, 116, 290-294.

(7) Decher, G.; Schmitt, J. Fine-tuning of the film thickness of ultrathin multilayer films composed of consecutively alternating layers of anionic and cationic polyelectrolytes. Progress in Colloid and Surface Science 1992, 89, 160-164.

(8) Lvov, Y.; Decher, G.; Möhwald, H. Assembly, Structural Characterization, and Thermal Behavior of Layer-by-Layer Deposited Ultrathin Films of Poly(vinyl sulfate) and Poly(allylamine). Langmuir 1993, 9, 481-486.

(9) Decher, G.; Lvov, Y.; Schmitt, J. Proof of multilayer structural organization in self-assembled polycation-polyanion molecular films. Thin Solid Films 1994, 244, $772-777$.

(10) Ostrander, J. W.; Mamedov, A. A.; Kotov, N. A. Two Modes of Linear Layer-byLayer Growth of Nanoparticle-Polyelectrolyte Multilayers and Different Interactions in the Layer-by-Layer Deposition. J. Am. Chem. Soc. 2001, 123, 1101-1110.

(11) Kleinfeld, E. R.; Ferguson, G. S. Stepwise Formation of Multilayered Nanostructural Films from Macromolecular Precursors. Science 2002, 265, 370373. 
(12) Correa-Duarte, M. A.; Giersig, M.; Kotov, N. A.; Liz-Marzán, L. M. Control of Packing Order of Self-Assembled Monolayers of Magnetite Nanoparticles with and without $\mathrm{SiO}_{2}$ Coating by Microwave Irradiation. Langmuir 1998, 14, 64306435.

(13) Grabar, K. C.; Freeman, R. G.; Hommer, M. B.; Natan, M. J. Preparation and Characterization of Au Colloidal Monolayers. Anal. Chem. 1995, 67, 735-743.

(14) Tillman, N.; Ulman, A.; Schildkraut, J. S.; Penner, T. L. Incorporation of Phenoxy Groups in Self-Assembled Monolayers of Trichlorosilane Derivatives: Effects on Film Thickness, Wettability, and Molecular Orientation. J.Am.Chem.Soc. 1988, 110, 6136-6144.

(15) Jiang, G.; Niederhauser, T. L.; Davis, S. D.; Lua, Y.-Y.; Cannon, B. R.; Dorff, M. J.; Howell, L. L.; Magleby, S. P.; Linford, M. R. Stability of Alkyl Monolayers on Chemomechanically Scribed Silicon to Air, Water, Hot Acid, and X-rays. Colloids and Surfaces A 2003, 226, 9-16.

(16) Tyrrell, J. W. G.; Attard, P. Images of Nanobubbles on Hydrophobic Surfaces and Their Interactions. Phys. Rev. Lett. 2001, 87, 176104/176101-176104/176104.

(17) Herzinger, C. M.; Johs, B.; McGahan, W. A.; Woollam, J. A.; Paulson, W. Ellipsometric determination of optical constants for silicon and thermally grown silicon dioxide via a multi-sample, multi-wavelength, multi-angle investigation. Journal of Applied Physics 1998, 83, 3323-3336.

(18) Handbook of Optical Constants of Solids; Academic Press: San Diego, 1998.

(19) Tompkins, H. G. A User's Guide to Ellipsometry; Academic Press: San Diego, 1993.

(20) Wacaser, B. A.; Maughan, M. J.; Mowat, I. A.; Niederhauser, T. L.; Linford, M. R.; Davis, R. C. Chemomechanical Surface Patterning and Functionalization of Silicon Surfaces Using an Atomic Force Microscope. Applied Physics Letters 2003, 82, 808-810. 


\section{APPENDIX}

CAD drawings of the accessories for the Gilson 215 liquid handler done in Pro-Engineer. 